\title{
$\angle S$ Research Suare

\section{Effect of Genetic Polymorphisms of CYP2C19 on the Steady-state Serum Concentrations of Valproic Acid in Chinese Han Patients With Schizophrenia}

\section{Shengdong WANG ( $\square$ wangsd0108@sina.com )}

Hangzhou Seventh People's Hospital https://orcid.org/0000-0003-4070-9623

Jing Li

Hangzhou Seventh People's Hospital

Mingfen Song

Hangzhou Seventh People's Hospital

Pan Yan

Hangzhou Seventh People's Hospital

Xuan Ju

Hangzhou Seventh People's Hospital

Jian Liu

Hangzhou Seventh People's Hospital

Chengpeng Wang

Hangzhou Seventh People's Hospital

Jianfei Shi

Hangzhou Seventh People's Hospital

Primary research

Keywords: valproic acid, CYP2C19, schizophrenia

Posted Date: July 20th, 2020

DOI: https://doi.org/10.21203/rs.3.rs-38955/v1

License: (c) (1) This work is licensed under a Creative Commons Attribution 4.0 International License.

Read Full License 


\section{Abstract}

Valproic acid is an anticonvulsant, which is also widely used for treating psychiatric disorders. Some clinical trials have demonstrated benefits of valproic acid augmentation therapy in schizophrenia. Interindividual variability in valproic acid dose and serum concentration may reflect functional consequences of genetic polymorphisms in genes encoding drug-metabolizing enzymes. The aim of this study was to determine the relationship between serum concentrations of valproic acid and single nucleotide polymorphisms of the cytochrome P450 (CYP) 2 C19 gene in patients with schizophrenia. All patients had been receiving fixed dose of valproic acid for at least 2 weeks. The daily doses were $0.5-$ $1.5 \mathrm{~g}$. No other drugs except olanzapine were coadministered. Serum concentrations of valproic acid were measured using the ultra-high performance liquid chromatography method with mass-spectrometric detection. The CYP2C19 (CYP2C19*2 G681A and CYP2C19*3 G636A) genotypes were identified by realtime PCR analyses. The mean concentration/dose ratios of valproic acid was significantly higher in patients with $1(P=0.029)$ or $2(P=0.007)$ mutated alleles for CYP2C19 than in those without mutated alleles. The mean concentration/dose ratios of valproic acid was significantly higher in patients with CYP $2 C 19 * 1 / 2$ genotype $(P=0.029)$ or $C Y P 2 C 19 * 2 / * 3$ genotype $(P=0.014)$ than in those with CYP2C12 ${ }^{*} 1 /{ }^{*} 1$ genotype. The findings of this study suggest that $C Y P 2 C 19$ genotypes play an important role in controlling steady-state serum concentrations of valproic acid in Chinese Han population.

\section{Introduction}

Over the last 40 years, a variety of adjunctive treatments have been used to treat schizophrenia (G. W. Christison et al., 1991). These are often used in addition to antipsychotics, in an attempt to alleviate the symptoms of schizophrenia such as hallucinations and delusional beliefs, although they have been used instead of antipsychotics. Valproate /valproic acid has been used for people whose psychosis did not respond to traditional therapy. Valproic acid is traditionally used as an anticonvulsant drug and is also used for affective disorders, especially for the treatment of acute mania (A. Cipriani et al., 2013). Furthermore it is thought to have anti-aggressive effects and it may reduce impulsive behaviour, which might be useful for some people with schizophrenia (L. Citrome et al., 2000). There is large interindividual variability in its pharmacokinetics and pharmacodynamics (Y. Ghodke-Puranik et al., 2013, J. Methaneethorn, 2018). Therefore, its serum concentration needs to be monitored as a guide of dose adjustment during the course of therapy.

The enzyme cytochrome P450 (CYP) 2 C19 is involved in the metabolism of valproic acid. Several mutated alleles of the CYP2C19 locus that cause decreased enzyme activity, that is, CYP2C19*2 (G681A, splicing defect) and CYP2C19*3 (G636A, W212X, premature stop codon) have been reported (K. Kurose et al., 2012, U. M. Zanger and M. Schwab, 2013). The steady-state serum concentration of valproic acid is significantly dependent on the mutated alleles. However, there are large overlaps in these steady-state serum concentrations among the different genotype groups and considerable interindividual variations in the values within each genotype. In previous published studies, body weight, age, gender and comedications had significant influence on VPA distribution volume (J. Ding et al., 2015, H. Nakashima et 
al., 2015, N. Ogusu et al., 2014). Although various genetic polymorphisms were associated with the increase or decrease of VPA serum concentration (T. Budi et al., 2015, X. M. Chu et al., 2012, W. Feng et al., 2016, Y. Guo et al., 2012, C. C. Hung et al., 2011, D. Jiang et al., 2009, T. K. Kiang et al., 2006, S. Mei et al., 2017, L. Tan et al., 2010), only two study identified the quantitative relationship between genetic polymorphisms CYP2C19 and VPA distribution volume in epileptic patients (D. Jiang et al, 2009, S. Mei et al., 2018).

Therefore, we aimed to investigate the effects of various factors including CYP2C19 polymorphisms, age, gender, body mass index (BMI) and duration of schizophrenia on the steady-state serum concentrations of valproic acid in Chinese Han patients with schizophrenia, which might be useful for VPA dose adjustment in clinical practice.

\section{Materials And Methods}

\section{Patients}

The subjects were 139 Chinese Han patients with schizophrenia (52 males and 87 females) who all fulfilled the criteria for schizophrenia, according to the ICD-10. They were physically healthy without any history of substance abuse, neurological disorder, delirium, or dementia and without any clinically significant findings, including a clinical laboratory examination, electrocardiography, and electroencephalography. The mean \pm SD of age and BMI value were $34.03 \pm 12.85$ years and $23.80 \pm 3.77$, respectively. 10 were smokers ( $\geq 10$ cigarettes/day), whereas the remainders were nonsmokers. None was a heavy drinker. This study was approved by the Ethics Committee of the Hangzhou $7^{\text {th }}$ people's hospital, and all the patients had given written informed consent to participate in this study.

\section{Drug treatment}

All the subjects had received valproic acid for at least 2 weeks. It has been shown that plasma concentrations of valproic acid reach steady state by 2 weeks after repeated oral administration. The daily dose was fixed and was given twice a day. The patients were receiving no drugs except olanzapine $(2.5-20 \mathrm{mg} / \mathrm{d})$. Female patients did not receive oral contraceptives. Patients' adherence was confirmed by the nursing staff or their families. Blood samples were taken at 8 AM.

\section{Analytical methods}

Serum concentrations of valproic acid were measured using the ultra-high performance liquid chromatography method with mass-spectrometric detection by Zhejiang BIOZON Medical Co.,Ltd. The lowest limit of detection for valproic acid was $2.5 \mu \mathrm{g} / \mathrm{ml}$ using $0.4 \mathrm{ml}$ of serum, and the interassay coefficient of variation was $7.98 \%$ at a concentration of $50 \mu \mathrm{g} / \mathrm{ml}$. The steady-state serum concentrations was adjusted by the doses of valproic acid, and the concentration/dose (C/D) ratios were used in statistical analyses. 
DNA was isolated from peripheral leucocytes using a nucleic acid extraction and purification kit (Kuangyuan, Suzhou, China). The *2 and *3 alleles of CYP2C19 were identified by PCR analyses using Human CYP2C19 genotyping kit (Kuangyuan, Suzhou, China).

\section{Statistical analyses}

The serum C/D ratios of valproic acid of patients were compared among different genotypic groups or different metabolic type groups using one way ANOVA. Multiple regression analyses were used to detect the correlation between serum C/D ratios of valproic acid and several factors, including the polymorphisms of CYP2C19, age, gender, BMI values, smoke and duration of schizophrenia. A P value of less than 0.05 was regarded as statistically significant. SSPS 19.0 for Windows was used for these statistical analyses.

\section{Results}

\section{Influence of CYP2C19 genotype on serumt C/D ratios of valproic acid}

To explore the effects of CYP2C19 genotype on interindividual variabilities in serum concentrations of valproic acid in Chinese Han schizophrenia, the CYP2C19*2 (G681A, splicing defect) and CYP2C19*3 (G636A, W212X, premature stop codon) were analyzed by real-time PCR. The characteristics of study population and the frequencies of CYP2C19 genotype are shown in Table 1. There was no significant differences in the general characteristics among genotypes.

Table 1

Characteristics of study population

\begin{tabular}{|llllllll|}
\hline & $* 1 /{ }_{1}$ & $* 2 / * 2$ & $* 3 / * 3$ & $* 1 / * 2$ & $* 1 / * 3$ & $* 2 / * 3$ & $p$ \\
\hline Male/female, $\mathrm{n}$ & $22 / 34$ & $5 / 9$ & $0 / 0$ & $21 / 37$ & $2 / 4$ & $2 / 3$ & 0.770 \\
Age, years & $34.55 \pm$ & $30.71 \pm$ & - & $34.66 \pm$ & $28.50 \pm$ & $35.60 \pm$ & 0.683 \\
& 12.39 & 9.98 & & 14.04 & 14.80 & 12.18 & \\
BMI & $23.58 \pm$ & $23.89 \pm$ & - & $23.69 \pm$ & $22.91 \pm$ & $23.88 \pm$ & 0.989 \\
& 3.31 & 2.63 & & 4.37 & 2.80 & 8.08 & \\
$\begin{array}{l}\text { Duration of } \\
\text { schizophrenia, } \\
\text { month }\end{array}$ & $123.29 \pm$ & $88.50 \pm$ & - & $126.55 \pm$ & $82.00 \pm$ & $182.40 \pm$ & 0.462 \\
\hline
\end{tabular}

Furthermore, associations between CYP2C19SNPs and serum C/D ratios of valproic acid were observed. The number of patients with CYP2C19*1/*1, $* 1 / * 2, * 1 / * 3, * 2 / * 3, * 2 / * 2$ and $* 3 / * 3$ genotype were 56,58 , $6,5,14$ and 0 , respectively (Table 2). Patients with CYP2C19 *1/*2 $(P=0.029)$ or CYP2C19*2/3 $(P=$ 0.014 ) had significantly higher serum C/D ratios of valproic acid than those with $C Y P 2 C 19{ }^{*} 1 /{ }^{*}$ (Table 2). And, the mean concentration/dose ratios of valproic acid was significantly higher in patients 
with 1 (heterozygous extensive metabolizers, $P=0.029$ ) or 2 (poor metabolizers, $P=0.007$ ) mutated alleles for CYP2C19 than in those without mutated alleles (Table 3 and Fig. 1).

Table 2

Steady-state serum C/D Ratio of VPA between

CYP2C19 genotypes

\begin{tabular}{|lcl|}
\hline Genotype & $\mathrm{N}$ & VPA C/D ratio $(\mu \mathrm{g} / \mathrm{ml} / \mathrm{g})$ \\
\hline$* 1 / * 1$ & 56 & $94.61 \pm 46.88$ \\
\hline$* 2 /{ }^{*} 2$ & 14 & $121.61 \pm 51.90$ \\
\hline$* 3 / * 3$ & 0 & - \\
\hline$* 1 / * 2$ & 58 & $114.61 \pm 47.57 \mathrm{a}$ \\
\hline$* 1 / * 3$ & 6 & $109.13 \pm 34.11$ \\
\hline$* 2 / * 3$ & 5 & $150.96 \pm 73.55 \mathrm{~b}$ \\
\hline a $p<0.05$ compared with $* 1 / * 1$ \\
\hline b $p<0.05$ compared with $* 1 / * 1$ \\
\hline
\end{tabular}

Table 3

Steady-state serum C/D Ratio of VPA between CYP2C19 metabolic types

\begin{tabular}{|c|c|c|}
\hline metabolic types & $\mathbf{N}$ & VPA C/D ratio $(\mu \mathrm{g} / \mathrm{ml} / \mathrm{g})$ \\
\hline Homozygous EMs (*1/*1) & 56 & $94.61 \pm 46.88$ \\
\hline heterozygous EMs $(* 1 / * 2 \nabla * 1 / * 3)$ & 64 & $114.10 \pm 46.29 \mathrm{a}$ \\
\hline $\mathrm{PMs}(* 2 / * 2 \rrbracket * 2 / * 3 \rrbracket * 3 / * 3)$ & 19 & $129.33 \pm 57.65 b$ \\
\hline \multicolumn{3}{|c|}{ a $p<0.05$ compared with Homozygous EMs } \\
\hline \multicolumn{3}{|c|}{ b $p<0.01$ compared with Homozygous EMs } \\
\hline EMs:extensive metabolizers; PMs & oor & etabolizers \\
\hline
\end{tabular}

\section{Multiple Regression Analysis}

Multiple regression analysis including CYP2C19 polymorphisms, age, gender, BMI values, smoke and duration of schizophrenia revealed that the polymorphisms of CYP2C19 (standardized beta $=0.251, \mathrm{p}<$ 0.01 ) and the BMI values (standardized beta $=-0.215, p<0.01$ ) were correlated with $C / D$ ratios of valproic acid (Table 4). 
Table 4

Standardized partial correlation coefficients

(Beta) between serum C/D ratio of VPA and various factors $(n=139)$

\begin{tabular}{|lll|}
\hline Variables & Beta & $\boldsymbol{p}$ \\
\hline CYP2C19 genotype & 0.251 & 0.003 \\
\hline Gender & -0.117 & 0.171 \\
\hline Age & 0.185 & 0.064 \\
\hline BMl & -0.215 & 0.009 \\
\hline Smoke & -0.130 & 0.135 \\
\hline Duration of Schizophrenia & -0.143 & 0.151 \\
\hline
\end{tabular}

\section{Discussion}

Genetic polymorphism may be an important source of interindividual variability in the pharmacokinetics and pharmacodynamics of valproic acid. In this study, the mean serum C/D ratios of valproic acid increased in accordance with the number of the mutated alleles for CYP2C19. It is the data that strongly implied that the mean serum C/D ratios of valproic acid increases in a gene dose-dependent manner. The mean concentration/dose ratios of valproic acid were significantly higher in patients with 1 or 2 mutated alleles for CYP2C19 than in those without mutated alleles. The result was assistant with previous report in epileptic (L. Citrome et al, 2000).

Otherwise, multiple regressin analyses showed that the serum C/D ratios of valproic acid were not only correlated with the number of mutated alleles for CYP2C19 but also associated with the BMI values. One study showed that loss-of-function CYP2C19 polymorphisms were associated with an increased BMI values (M. Noai et al., 2016). This may explained the association between BMI values and the serum C/D ratios of valproic acid in our study. And some studies reported the effect of age on the metabolism of valproic acid (U. A. Argikar and R. P. Remmel, 2009, S. J. Miyagi and A. C. Collier, 2011), showed that there was no statistically significant difference in the rate of VPA metabolism. Another study showed that serum valproic acid levels was significantly increased with younger age (L. Ben Mahmoud et al., 2017). These results are inconsistent. A larger population sample size is needed to further verify the results.

This study has several limitations. The dose of valproic acid was not unified. Data of clinical responses related with the observed differences in the steady-state serum concentrations of valproic acid were lacking. The possibility of committing type II errors cannot be ruled out because of the low power.

\section{Conclusions}


The findings of this study suggest that CYP2C19 genotypes play an important role in controlling the steady-state serum concentrations of valproic acid in Chinese Han subjects.

\section{Declarations}

\section{Ethics approval and consent to participate}

The study was approved by the Ethics Committee of the Hangzhou Seventh People's Hospital. The purpose and importance of the study were explained to each participant before they proceeded into actual activities. Informed consent was obtained from all participants.

\section{Consent for publication}

Not applicable.

\section{Availability of data and material}

All data generated or analyzed during this study are included in this published article.

\section{Competing interests}

There are no conflicts of interest to report for any of the authors.

\section{Funding}

Funding for this study was provided by Grant 2015C03054 from the Key Research Project of the Zhejiang Province, Science and Technology Project of Medicine and Health of Zhejiang Province of China (2020KY222) and Zhejiang Provincial Natural Science Foundation of China (LY17H090013).

\section{Authors' contributions}

Wang S, Song M, Shi J were responsible for study design, Ju X, Liu J, Wang C, Shi J were responsible for recruiting the subjects, Wang S, Li J, Yan P, Ju X were responsible for collecting clinical data and performing the clinical rating. Wang S, Li J, Yan $\mathrm{P}$ were responsible for statistical analysis, and manuscript. All authors contributed to and have approved the final manuscript.

\section{Acknowledgements}

We thank the patients who participate in our study. We are grateful to the funding of this study.

\section{References}

1. Argikar, U. A. and R. P. Remmel. 2009. "Effect of Aging on Glucuronidation of Valproic Acid in Human Liver Microsomes and the Role of Udp-Glucuronosyltransferase Ugt1a4, Ugt1a8, and Ugt1a10." Drug Metab Dispos, 37(1), 229-36. 
2. Ben Mahmoud, L.; A. Hakim; H. Ghozzi; R. Atheymen; Z. Sahnoun and K. Zeghal. 2017. "Influence of Age and Co-Medication on the Steady-State Pharmacokinetics of Valproic Acid in Tunisian Patients with Epilepsy." Rev Neurol (Paris), 173(3), 159-63.

3. Budi, T.; K. Toth; A. Nagy; Z. Szever; A. Kiss; M. Temesvari; E. Hafra; M. Garami; A. Tapodi and K. Monostory. 2015. "Clinical Significance of Cyp2c9-Status Guided Valproic Acid Therapy in Children." Epilepsia, 56(6), 849-55.

4. Christison, G. W.; D. G. Kirch and R. J. Wyatt. 1991. "When Symptoms Persist: Choosing among Alternative Somatic Treatments for Schizophrenia." Schizophr Bull, 17(2), 217-45.

5. Chu, X. M.; L. F. Zhang; G. J. Wang; S. N. Zhang; J. H. Zhou and H. P. Hao. 2012. "Influence of UdpGlucuronosyltransferase Polymorphisms on Valproic Acid Pharmacokinetics in Chinese Epilepsy Patients." Eur J Clin Pharmacol, 68(10), 1395-401.

6. Cipriani, A.; K. Reid; A. H. Young; K. Macritchie and J. Geddes. 2013. "Valproic Acid, Valproate and Divalproex in the Maintenance Treatment of Bipolar Disorder." Cochrane Database Syst Rev, (10), CD003196.

7. Citrome, L.; J. Levine and B. Allingham. 2000. "Changes in Use of Valproate and Other Mood Stabilizers for Patients with Schizophrenia from 1994 to 1998." Psychiatr Serv, 51(5), 634-8.

8. Ding, J.; Y. Wang; W. Lin; C. Wang; L. Zhao; X. Li; Z. Zhao; L. Miao and Z. Jiao. 2015. "A Population Pharmacokinetic Model of Valproic Acid in Pediatric Patients with Epilepsy: A Non-Linear Pharmacokinetic Model Based on Protein-Binding Saturation." Clin Pharmacokinet, 54(3), 305-17.

9. Feng, W.; S. Mei; L. Zhu; Y. Yu; W. Yang; B. Gao; X. Wu; Z. Zhao and F. Fang. 2016. "Effects of Ugt1a6 and Gabra1 on Standardized Valproic Acid Plasma Concentrations and Treatment Effect in Children with Epilepsy in China." Ther Drug Monit, 38(6), 738-43.

10. Ghodke-Puranik, Y.; C. F. Thorn; J. K. Lamba; J. S. Leeder; W. Song; A. K. Birnbaum; R. B. Altman and T. E. Klein. 2013. "Valproic Acid Pathway: Pharmacokinetics and Pharmacodynamics." Pharmacogenet Genomics, 23(4), 236-41.

11. Guo, Y.; C. Hu; X. He; F. Qiu and L. Zhao. 2012. "Effects of Ugt1a6, Ugt2b7, and Cyp2c9 Genotypes on Plasma Concentrations of Valproic Acid in Chinese Children with Epilepsy." Drug Metab Pharmacokinet, 27(5), 536-42.

12. Hung, C. C.; J. L. Ho; W. L. Chang; J. J. Tai; T. J. Hsieh; Y. W. Hsieh and H. H. Liou. 2011. "Association of Genetic Variants in Six Candidate Genes with Valproic Acid Therapy Optimization." Pharmacogenomics, 12(8), 1107-17.

13. Jiang, D.; X. Bai; Q. Zhang; W. Lu; Y. Wang; L. Li and M. Muller. 2009. "Effects of Cyp2c19 and Cyp2c9 Genotypes on Pharmacokinetic Variability of Valproic Acid in Chinese Epileptic Patients: Nonlinear Mixed-Effect Modeling." Eur J Clin Pharmacol, 65(12), 1187-93.

14. Kiang, T. K.; P. C. Ho; M. R. Anari; V. Tong; F. S. Abbott and T. K. Chang. 2006. "Contribution of Cyp2c9, Cyp2a6, and Cyp2b6 to Valproic Acid Metabolism in Hepatic Microsomes from Individuals with the Cyp2c9*1/*1 Genotype." Toxicol Sci, 94(2), 261-71. 
15. Kurose, K.; E. Sugiyama and Y. Saito. 2012. "Population Differences in Major Functional Polymorphisms of Pharmacokinetics/Pharmacodynamics-Related Genes in Eastern Asians and Europeans: Implications in the Clinical Trials for Novel Drug Development." Drug Metab Pharmacokinet, 27(1), 9-54.

16. Mei, S.; W. Feng; L. Zhu; X. Li; Y. Yu; W. Yang; B. Gao; X. Wu; F. Fang and Z. Zhao. 2018. "Effect of Cyp2c19, Ugt1a8, and Ugt2b7 on Valproic Acid Clearance in Children with Epilepsy: A Population Pharmacokinetic Model." Eur J Clin Pharmacol, 74(8), 1029-36.

17. Mei, S.; W. Feng; L. Zhu; Y. Yu; W. Yang; B. Gao; X. Wu; Z. Zhao and F. Fang. 2017. "Genetic Polymorphisms and Valproic Acid Plasma Concentration in Children with Epilepsy on Valproic Acid Monotherapy." Seizure, 51, 22-26.

18. Methaneethorn, J. 2018. "A Systematic Review of Population Pharmacokinetics of Valproic Acid." Br $\mathrm{J}$ Clin Pharmacol, 84(5), 816-34.

19. Miyagi, S. J. and A. C. Collier. 2011. "The Development of Udp-Glucuronosyltransferases 1a1 and 1a6 in the Pediatric Liver." Drug Metab Dispos, 39(5), 912-9.

20. Nakashima, H.; K. Oniki; M. Nishimura; N. Ogusu; M. Shimomasuda; T. Ono; K. Matsuda; N. YasuiFurukori; K. Nakagawa; T. Ishitsu, et al. 2015. "Determination of the Optimal Concentration of Valproic Acid in Patients with Epilepsy: A Population Pharmacokinetic-Pharmacodynamic Analysis." PLoS One, 10(10), e0141266.

21. Noai, M.; H. Soraoka; A. Kajiwara; Y. Tanamachi; K. Oniki; K. Nakagawa; T. Ishitsu and J. Saruwatari. 2016. "Cytochrome P450 2c19 Polymorphisms and Valproic Acid-Induced Weight Gain." Acta Neurol Scand, 133(3), 216-23.

22. Ogusu, N.; J. Saruwatari; H. Nakashima; M. Noai; M. Nishimura; M. Deguchi; K. Oniki; N. YasuiFurukori; S. Kaneko; T. Ishitsu, et al. 2014. "Impact of the Superoxide Dismutase 2 Val16ala Polymorphism on the Relationship between Valproic Acid Exposure and Elevation of GammaGlutamyltransferase in Patients with Epilepsy: A Population Pharmacokinetic-Pharmacodynamic Analysis." PLoS One, 9(11), e111066.

23. Tan, L.; J. T. Yu; Y. P. Sun; J. R. Ou; J. H. Song and Y. Yu. 2010. "The Influence of Cytochrome Oxidase Cyp2a6, Cyp2b6, and Cyp2c9 Polymorphisms on the Plasma Concentrations of Valproic Acid in Epileptic Patients." Clin Neurol Neurosurg, 112(4), 320-3.

24. Zanger, U. M. and M. Schwab. 2013. "Cytochrome P450 Enzymes in Drug Metabolism: Regulation of Gene Expression, Enzyme Activities, and Impact of Genetic Variation." Pharmacol Ther, 138(1), 10341.

\section{Figures}




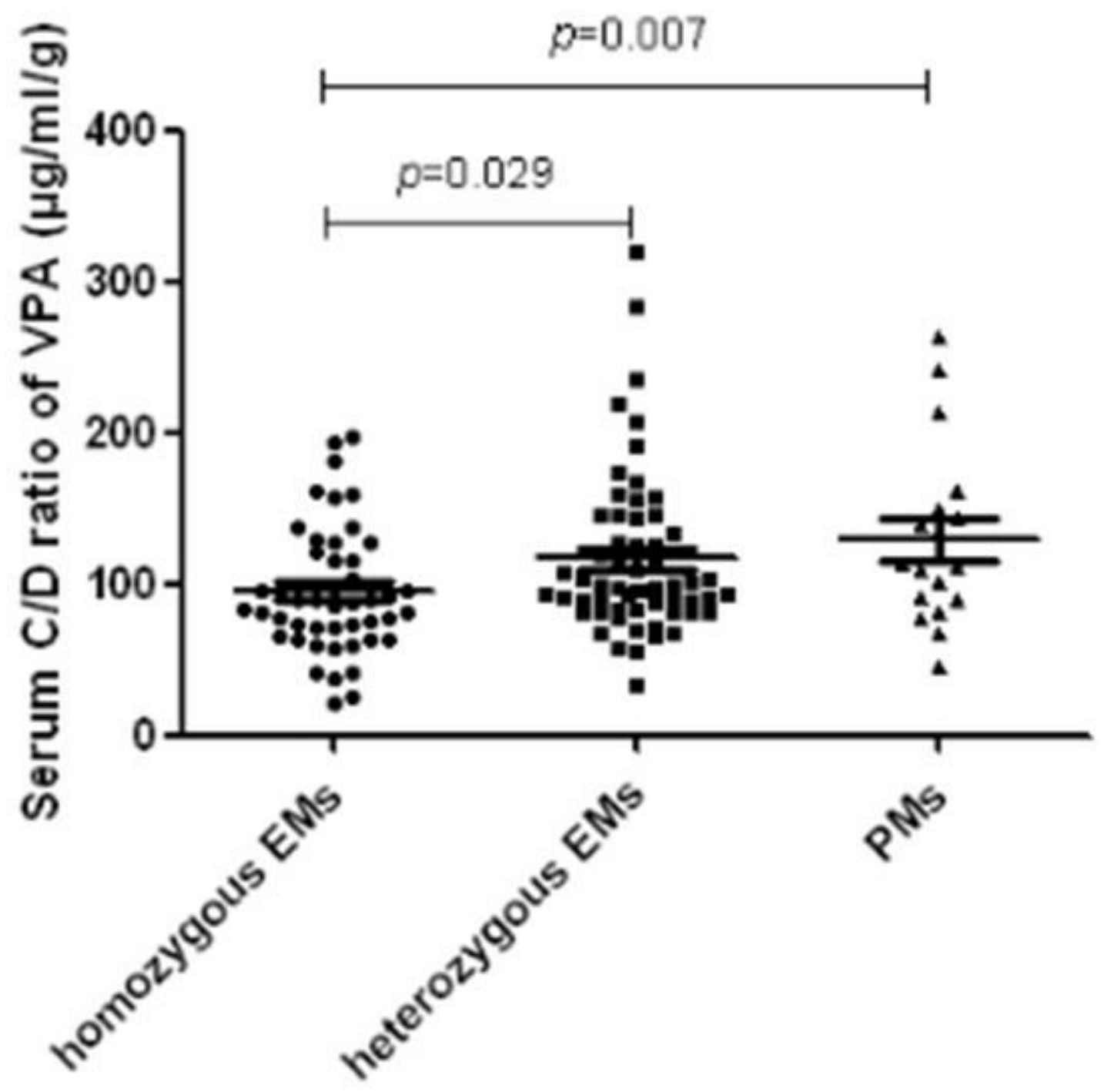

Figure 1

Differences in serum concentration/dose ratio of valproic acid in various metabolizer of CYP2C19 in Chinese schizophrenia patients. EMs: extensive metabolizers; PMs: poor metabolizers. 